\title{
Fingerprint Core Point Detection Algorithm Using Orientation Field Based Multiple Features
}

\author{
H B Kekre \\ Department of Computer Science \\ Mukesh Patel School of Technology Management \& \\ Engineering, NMIMS University \\ Mumbai, India
}

\author{
$\checkmark$ A Bharadi \\ Department of Computer Science \\ Mukesh Patel School of Technology Management \& \\ Engineering, NMIMS University \\ Mumbai, India
}

\begin{abstract}
Fingerprint recognition is a widely used biometric identification mechanism. In case of correlation based fingerprint recognition detection of a consistent registration point is a crucial issue; this point can be a core point of a fingerprint. Many techniques have been proposed but success rate is highly dependent on input used and accurate core point detection is still an open issue. Here we discuss an algorithm which is based on multiple features derived from the fingerprint which are collectively used for consistent core point detection. Here we use Orientation field, coherence, Poincare index for core point detection. Though all fingerprints don't possess core point still this algorithm is useful to detect high curvature regions and gives high accuracy as it combines advantages form individual features. This algorithm is crucial in the development of correlation based Automatic Fingerprint Recognition System (AFIS).
\end{abstract}

\section{Categories and Subject Descriptors}

I.4.7 Image Processing and Computer vision

\section{General Terms}

Algorithms, Experimentation, Security, Verification.

\section{Keywords}

Fingerprint Recognition, Core Point, Orientation.

\section{INTRODUCTION}

Fingerprints are the oldest and most widely recognized biometric trait. All human being posses fingerprint and these fingerprints are result of unique ridge and valley structure formed by skin over the fingers [18]. Ridges and valleys are often run in parallel; these structures have bifurcation and ridge endings called as termination. The ridge structure as a whole takes different shapes, characterized by high curvature, terminations, bifurcations, crossover etc. These regions are called singularity.

These singularities may be classified into three topologies; loop, delta and whorl. What makes fingerprint unique is the distribution of such structures at local level. These are called as minutiae [10]. Minutiae mean small details and this refers to the various ways that the ridges can be discontinuous. A sudden ridge end is called termination or it can divide into two ridges which is called bifurcations. Figure 1 shows a fingerprint image.

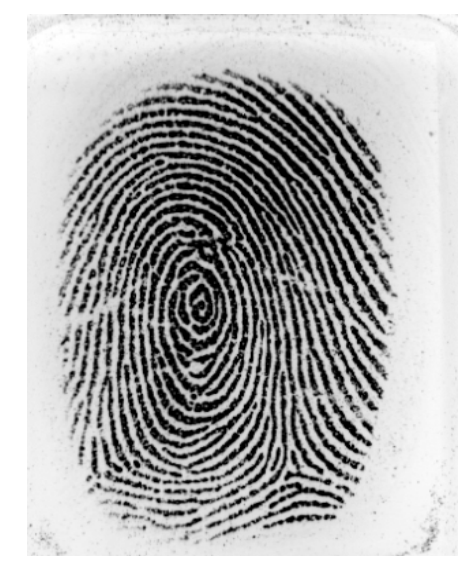

Figure.1. Typical fingerprint as scanned by optical fingerprint scanner

Closer observation of such image can give us the location of present minutiae. Some of typical structures are shown in Fig. 2. We can see a ridge ending in Figure 2 (a), a core point in Figure 2 (b). Figure 2 (c) shows a ridge bifurcation. A loop present on a ridge is shown in Figure 2 (d).

Automatic Fingerprint Identification Systems (AFIS) try to match fingerprint by matching these ridge valley structure. Mainly two types are systems are there (a) Minutiae based matching (b) Correlation based matching Minutiae based system try to identify the location and type of minutia and match it with database template. The accuracy is totally dependent on the identification of minutia point [14][1][8]. In case of correlation based techniques, rather than detecting minutiae, we go for global matching of ridge valley structure, here we try to match the texture of fingerprint. Such techniques are robust but less accurate $[2][11][12]$. 


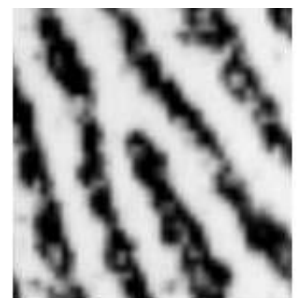
(a)

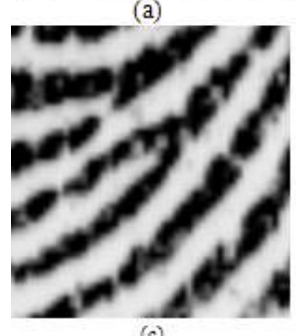

(c)

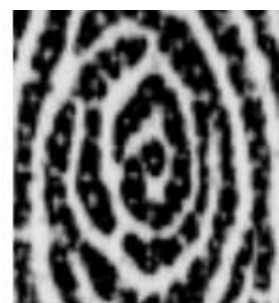

(b)

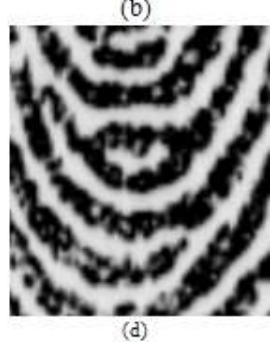

Fig. 2 Typical Minutiae structures (a) Ridge ending (b) A core point. (c) Ridge bifurcation. (d) A loop present on a ridge

\subsection{Correlation based Fingerprint Recognition}

As discussed earlier the core point based techniques are robust, but for matching the global ridge structure, we need a consistent point for aligning the fingerprints. This point is called as Registration Point, the fingerprints have various ridge structures present on them, out of which the core points can be detected and used as Registration Point. In case of the fingerprint which don't have core point we go for detection of point with high curvature regions or Low Coherence Strength. An example of Core point is shown in Figure 3.
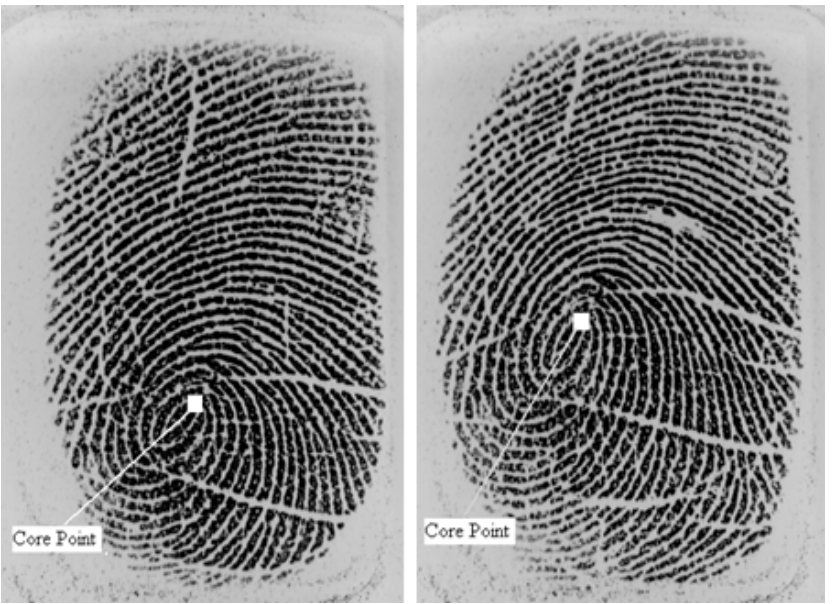

Figure.3. Two fingerprints of same finger showing the core point.

In [4] authors have described a fingerprint matching system based on Gabor filters, which uses circular tessellation around the core point and extracts Gabor Magnitude in 8 Directions, this is used as a feature vector, similar approach which uses a filter bank of Gabor filters is proposed in [8], they are using a set of filters to extract the fingerprint feature vectors and this is used for the training of classifier. In [2] Cavusoglu A. et al. have proposed a robust approach which operates on grey levels of fingerprint and calculates global feature vector taking registration point at the reference. Success all these approaches is based on accurate determination of the core point (Registration Point). They have used various methods for determining the core point, which are discussed in the next section.

\section{EXISTING CORE POINT DETECTION MECHANISM}

Core point detection is a non trivial task. To determine the location of the core point we first need to estimate the orientation field of the fingerprint. Various approaches available in the literature, In [5] Hong, Jain and Bolle has used a Hierarchical method based on fingerprint gradient and its consistency level, Another approach using gradient and continuous vector field proposed in [6] by Hong and Jain. In [17] Wang Y., Hu J., Han F have proposed an Enhanced Gradient field based algorithm to obtain smoother field. We have developed an algorithm based on gradient and neighborhood field this algorithm is based on neighborhood field averaging [9] gives faster and smoother field estimate; the orientation field angles are in the range of 0 to 180 degrees. The typical orientation field of a fingerprint in Figure 1. is shown below for a block size of $16 \times 16$,

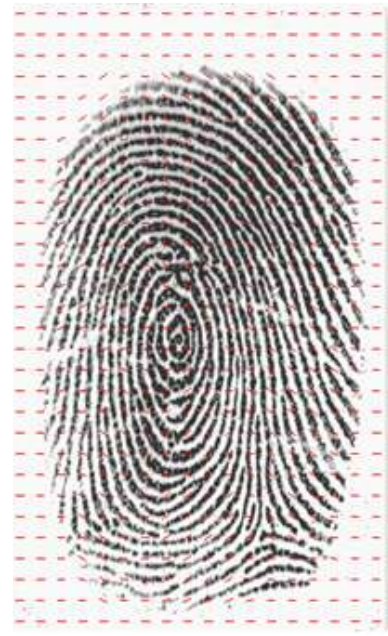

(a)

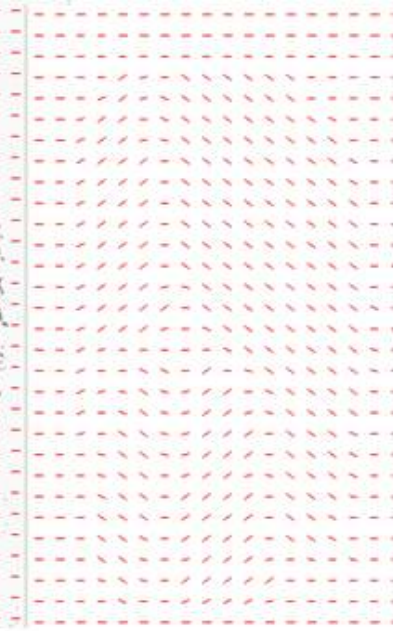

(b)
Figure 4. Fingerprint Orientation Map (a) Orientation Field mapped over Fingerprint (b) Orientation Field Map

The core point detection is based on the orientation field calculation as all the methods are using the orientation field information to detect the core point. Another parameter is also used in this calculation which is called as coherence level [17][9] or field strength. We discuss the existing Core point detection algorithms in the coming section.

\subsection{Core Point Detection using Integration of Sine Component of the Fingerprint Orientation [8] [2]}

In this method the sine component of the orientation filed is integrated in a semi circular region, with three segments and the Components are linearly summed up in a specific manner as discussed in [14], this method give a good approximation of 
fingerprint but accuracy is still low, and for better approximation more number of iterations are required. The regions for integration are RI, and RII, the sine component $\sin (\mathrm{o}(\mathrm{i}, \mathrm{j}))$ of these regions are integrated and final field $A(i, j)$ is given by

$$
A(i, j)=\sum_{R I} \sin (O(i, j))-\sum_{R I I} \sin (O(i, j))
$$

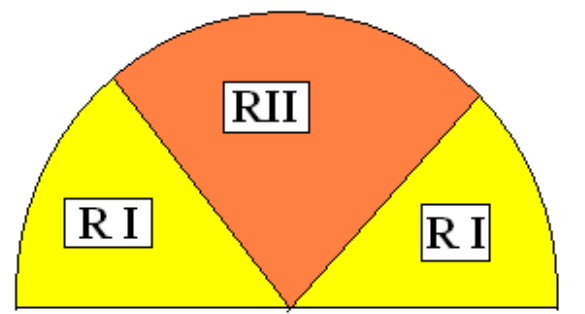

(i,j)

Figure 4. Regions for integrating sine component of orientation field to estimate field at $(\mathbf{i}, \mathbf{j})$

\subsection{Core Point Estimation using Poincare Index $[8][14]$}

This approach is based on calculation of Poincare index of all the points in orientation map, we actually determine the Poincare index by calculating the consecutive points field angle difference and summing it, the point enclosed by a digital curve (Core Point) will have highest Poincare index. The Poincare Index map is then thresholded and the point with highest value is taken as core point. This method is also used in [1] by Afsar F. et al. This method is also recursive, if no core point is found then the orientation field is smoothened and again the same procedure is followed. If still core point is not estimated then the authors have suggested a covariance based method, but this is computationally expensive.

\subsection{Core Point Estimation using Direction Codes and Curve Classification [3]}

In this method first the orientation field is calculated, from this field the directional codes are generated. The direction codes are used for rough estimate and a sampled matrix is generated. This matrix and curve classification method similar to chain codes is used for accurate core point detection. This method requires more steps and the procedure given in this paper is not suitable for arch type prints, since it is not possible to define the core point in this case.

\subsection{Core Point Detection using Squared Orientation Image \& Complex Convolution Masks [15]}

S. Chikkerur, N. Ratha have used Complex convolution map for core and delta points over the squared orientation field to obtain the core and delta point in the fingerprint. This method covers detection of core as well as delta points, and it is a multistep process. The accuracy obtained is good around $95-98 \%$. At the same time the mathematical complexity is high and the method needs post processing steps also.
We have implemented Poincare based method and sine component integration based methods, the achieved detection rate were moderate, this is mainly because the different database used and the block size used for the orientation field estimation.

The core point detection mechanism can still be improved for simplicity and to give better results. Here we have proposed a scheme which combines the advantages of above discussed features. We discuss this method in the next section.

\section{PROPOSED TECHNIQUE}

The proposed technique is based on multiple features extracted from a fingerprint the feature set includes

1. Coherence of grayscale gradient

2. Poincare index

3. Angular Coherence

4. Orientation Field Mask

\subsection{Coherence of Gradient [17]}

Coherence is the Field strength of the gradient. The image is divided into WXW size blocks and we find $\mathrm{X}$ and $\mathrm{Y}$ directional gradient operator like $3 * 3$ Sobel operator or another choice is to use a complex Marr-Hilderth operator. In this paper we have calculated gradient using a $3 * 3$ Sobel mask for horizontal and vertical gradient calculation. Actually as shown in Fig. 4 the ridges may have two edges in a selected block, and hence the calculated gradient vectors at both sides of ridge are opposite to each other, the opposite gradients at both sides of a ridge are likely to cancel each other. To solve this problem we use a method used in [17], involves doubling the gradient angles before the averaging. This method involves calculation of squared gradient. The gradient vectors can be denoted as $\left[g_{x}, g_{y}\right]^{T}$. Because of the doubling process the gradient angle $(\varphi+\pi)$ becomes $(2 \varphi+2 \pi)$. Practically $2 \varphi$ is the angle of squared gradient vectors $\left[g_{s x}, g_{s y}\right]^{T}$ that has the following relation with $\left[\mathrm{g}_{\mathrm{x}}, \mathrm{g}_{\mathrm{y}}\right]^{\mathrm{T}}$, hence we can write using trigonometric identities,

$$
\left(\begin{array}{l}
g_{s x} \\
g_{s y}
\end{array}\right)=\left(\begin{array}{l}
g^{2} \cos 2 \varphi \\
g^{2} \sin 2 \varphi
\end{array}\right)=\left(\begin{array}{c}
g^{2}\left(\cos ^{2} \varphi-\sin ^{2} \varphi\right) \\
g^{2}(2 \sin \varphi-\cos \varphi)
\end{array}\right)=\left(\begin{array}{c}
g_{x}^{2} \\
2 g_{x} g_{y}
\end{array}\right)
$$

The averaged squared gradients $\left[\overline{g_{s x}}, \overline{g_{s y}}\right]$ in a block of size $\mathrm{W}^{*} \mathrm{~W}$ can therefore be calculated by

$$
\left(\begin{array}{l}
\overline{g_{s x}} \\
g_{s y}
\end{array}\right)=\left(\begin{array}{c}
\sum_{W} g_{s x} \\
\sum_{W} g_{s y}
\end{array}\right)=\left(\begin{array}{c}
\sum_{W}\left(g_{x}^{2}-g_{y}^{2}\right) \\
\sum_{W} 2 g_{x} g_{y}
\end{array}\right)
$$

We divide the input fingerprint image into equal size blocks of $\mathrm{W}$ $\mathrm{X} \mathrm{W}$ pixels, and average over each block independently. In this paper we are interested in coherence], which indicates strength of the averaged gradients, coherence is given by 


$$
\operatorname{Coh}_{W}=\frac{\left|\sum_{i=1}^{W} \sum_{i=1}^{W}\left(g_{s x}(i, j) \llbracket g_{s y}(i, j)\right)\right|}{\sum_{i=1}^{W} \sum_{i=1}^{W}\left|g_{s x}(i, j) \llbracket g_{s y}(i, j)\right|}
$$

The coherence value is low at the high curvature regions, the core points are mainly high curvature regions hence this property is very useful to find the location of core point. We use the coherence map to calculate neighborhood averaged coherence, in a region of $3 \times 3$ block neighborhood, this gives smoother coherence map. The actual coherence and averaged coherence is shown in Figure 5. Red region indicate high coherence, yellow and blue region indicate decreasing coherence. The regions around core point have low value.

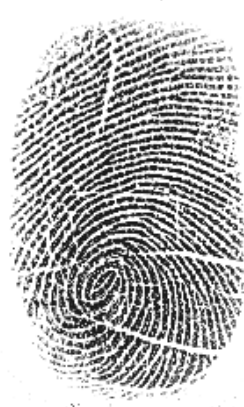

(a)

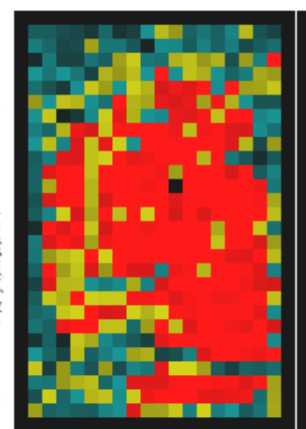

(b)

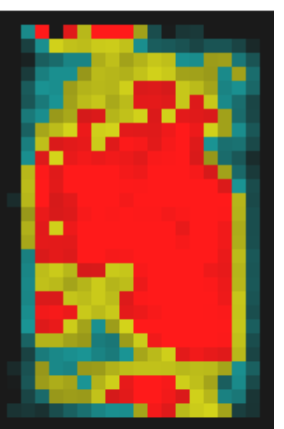

(c)
Figure 5. (a) Original Fingerprint (b) Coherence Map (c) Neighborhood Averaged Coherence Map

\subsection{Poincare Index [8][14]}

This index gives presence of core point by evaluating orientation angle over a closed digital curve. We first detect presence of core point using the unique mask that operates on orientation field and then find Poincare index in the selected 7X7 Blocksize. The Poincare index is high at high curvature point or the core, the map of Poincare index at selected part is shown below, this map is used to improve accuracy of core point detection.

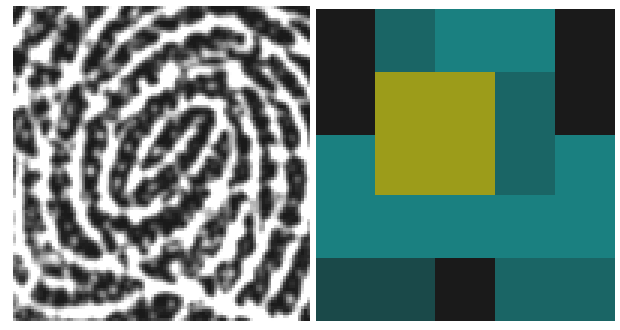

Figure 6. Selected region and corresponding Poincare index Map, yellow region indicate High Poincare index

\subsection{Angular Coherence [16]}

We sum cosine component of difference between orientation angle of center block and the neighborhood of 5X5 blocks of orientation image, the sum of cosine component is low at the core point as the difference is high and the normal region where the orientation is uniform the sum is high as all blocks tend to have similar orientation. $\mathrm{O}(\mathrm{Cx}, \mathrm{Cy})$ is the block under consideration $\mathrm{O}(\mathrm{i}, \mathrm{j})$ is the block in the $5 \mathrm{X} 5$ neighborhood.

$$
C=\sum_{W X W} \cos ((o(C x, C y)-o(i, j))
$$

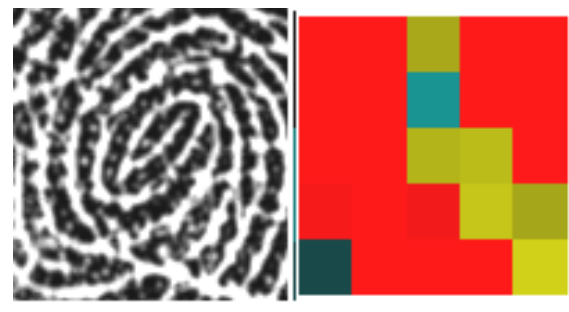

Figure 7. Cosine component of angular difference, Blue Square indicate minimum value in the region.

\subsection{Orientation Field Mask}

This feature is used to locate the core point region. This method is based on the fact that the core points are having specific pattern of the orientation field, the patterns appear like a loop formed in the region of core point. We are using a mask which gives maximum magnitude of convolution in the region of core point. An example of orientation field in the region of core point is shown in Fig. 8

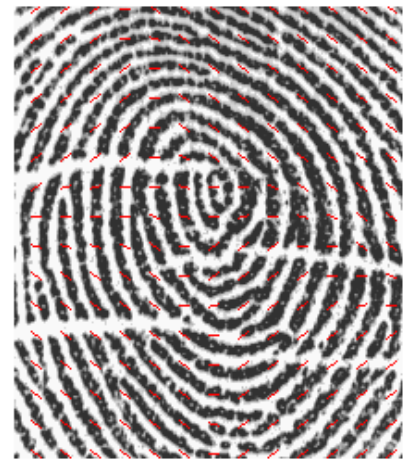

(a)

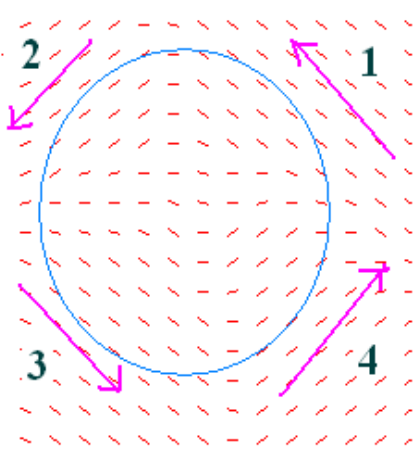

(b)
Figure 8. Orientation Field at the core point (a) Core Point (b) Loop Formed by the orientation field.

The angle in the region 1,3 have range of (45 to 90 degrees) and the angles in the regions have range of ( 0 to 45 degrees). If any such pattern exists in a fingerprint it can be treated as a core point. We are using a mask which is empirically generated to locate such pattern in the orientation field and convolution with the orientation map gives maximum response at the location of core point or loop type regions. The mask is a $9 \times 9$ dimensional array shown below in Figure 9. After applying this mask at each element of the orientation map we get a magnitude which corresponds to the nature of orientation field around that point for a point $\mathrm{O}(\mathrm{i}, \mathrm{j})$ in the orientation loop field this field strength is given by 
Loop Field Strength $=$

$$
\begin{aligned}
& \sum_{x=-W / 2}^{x=W / 2} \sum_{y=-W / 2}^{y=W / 2} O M[x, y] \sqcap O[i+x, j+y] \\
& O M=\left[\begin{array}{ccccccccc}
-1 & -1 & -1 & -1 & 0 & +1 & +1 & +1 & +1 \\
-1 & -1 & -1 & -1 & 0 & +1 & +1 & +1 & +1 \\
-1 & -1 & -1 & -1 & 0 & +1 & +1 & +1 & +1 \\
-1 & -1 & -1 & -1 & 0 & +1 & +1 & +1 & +1 \\
0 & 0 & 0 & 0 & 0 & 0 & 0 & 0 & 0 \\
+1 & +1 & +1 & +1 & 0 & -1 & -1 & -1 & -1 \\
+1 & +1 & +1 & +1 & 0 & -1 & -1 & -1 & -1 \\
+1 & +1 & +1 & +1 & 0 & -1 & -1 & -1 & -1 \\
+1 & +1 & +1 & +1 & 0 & -1 & -1 & -1 & -1
\end{array}\right]
\end{aligned}
$$

Figure 9. Orientation Field mask

Where $\mathrm{W}$ is the mask size ( $\mathrm{W}=9$ in our case), we have developed this unique mask after observation of orientation fields of various fingerprints, this mask efficiently locates a complete loop if present in a fingerprint and locates the region of high curvature in case of the fingerprints which don't have core point. The loop field strength is calculated for each element in the orientation map and next step is to threshold this magnitude array.

\subsection{Proposed Core Point Detection Algorithm}

Considering all above discussed features, we propose a formal algorithm which combines them to estimate the core point location. The core point detection mechanism proposed works in two main stages.

1. Detect possible core point (Singularity) region. We mainly consider Finger core but the mask used gives presence of Arch in the form of high curvature ridges also.

2. Detect a single point in the region by weighted sum of feature vectors discussed above. This step decides presence of core point as a center of a 16X16 Pixels window.

The algorithm for core point detection is given as follows:

1. Read the fingerprint Image

2. Preprocess \& Segment the fingerprint image to remove errors due to non uniform pressure and illumination while scanning of the fingerprint.

3. Estimate the orientation field by optimized neighborhood averaging.

4. Calculate the Gradient Field Coherence, Poincare index, Angular Coherence features \& normalize these feature vectors to a 0 to 1 range.

5. Calculate loop field Strength at each point in the orientation field, using the developed orientation field mask.
6. Normalize the loop field strength array in a range of 0 to 1 .

7. Threshold the loop field strength array to locate the core point, this threshold for our method ranges in the order of $(.34$ to .45$)$. Take the centroid of the region if it consists more than one block.

8. If more than one core points regions are located then take the region towards upper end or take centroid.

9. Separate $5 \times 5$ block size (Each block of size $16 \times 16$ Pixels considered here) area of the Fingerprint \& its corresponding parameters.

10. Using These Parameters determine the exact core point location by weighted sum as discussed below.

This first stage (Step 1 to 9) give the core point region as shown below in Figure 10. We copy the region into a $5 \mathrm{X} 5$ size array and evaluate above discussed features for the regions

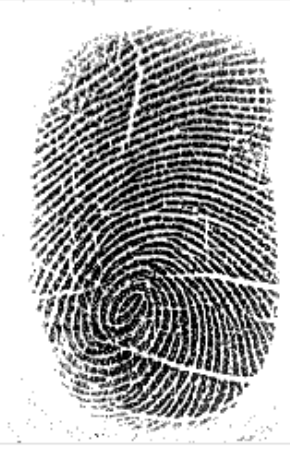

(a)

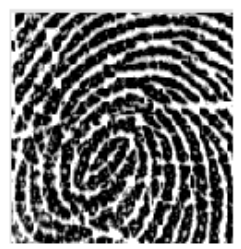

(c)

(d)

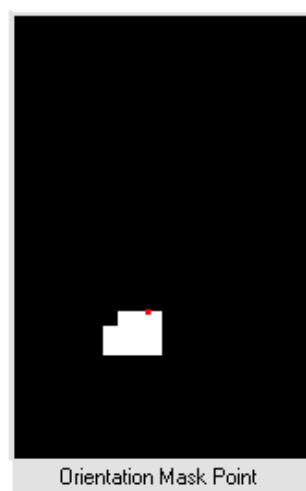

(b)

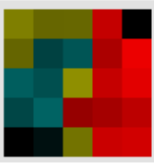

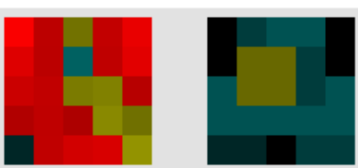

(e) (f)
Fig. 9 (a) Selected Fingerprint (b) Thresholded Orientation Point Mask Output (c) Selected Core Regopn (d) Coherence Feature for 5X5 Region (b) Angular Coherence Feature (d) Poincare Index Feature .

Final core point is decided by weighted sum of the above parameters for core point the coherence $\&$ cosine component sum should be minimum and Poincare index should be maximum, hence we find final region weighted sum as

$$
\begin{aligned}
\text { Core }[\mathrm{x}, \mathrm{y}]= & \text { Coherence }[\mathrm{x}, \mathrm{y}]+\text { Angular Coherence }[\mathrm{x}, \mathrm{y}]- \\
& \text { Poincare }[\mathrm{x}, \mathrm{y}]
\end{aligned}
$$

This gives final region is shown in Fig. 11. The final region ma is shown as core point region, we select the minimum value from this map as the core point block, the red block in the final map shows selected core point in Fig 11 (c). Fig 11(a) shows the Feature vectors and final Core point block. 


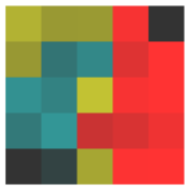

Coherence Core

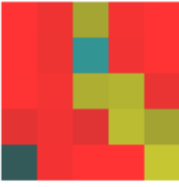

Angle Core

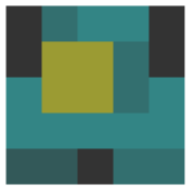

Poincare Core

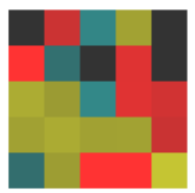

CorePoint Region

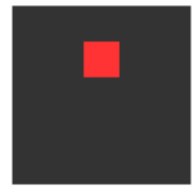

Final Core Region

(a)

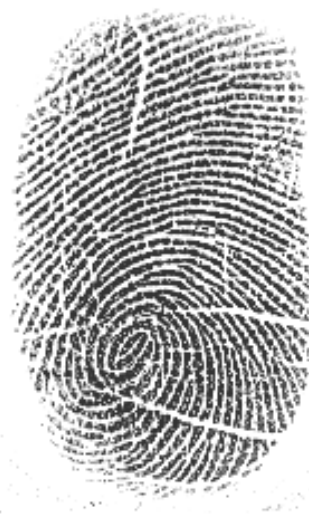

(b)

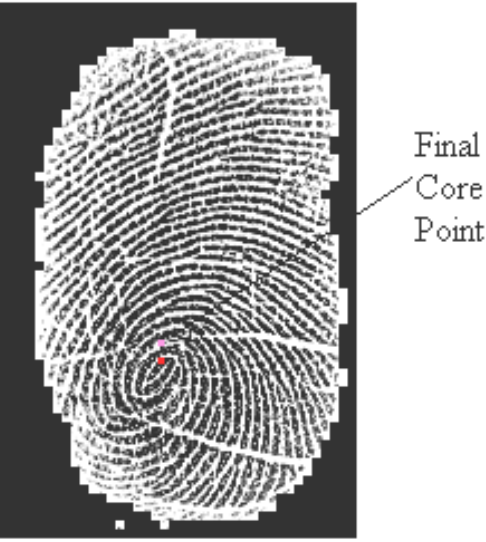

(c)

Figure 11 (a) Feature Vectors (b) Selected Fingerprint (c) Fingerprint with marked core point .

\section{RESULTS}

This algorithm is implemented on MS Visual Studio 2005 platform, using Visual C\# 2.0. The program was tested on AMD Athlon 64 processor running at $1.8 \mathrm{GHZ}$ with $1.5 \mathrm{~GB}$ DDRII RAM, operating system is Windows XP SP3. Block size considered is $16 \times 16$ pixels. For testing purpose we have used FVC 2000, 2002 \& 2004 Databases [4], we have also used optical fingerprint scanner Futronics FS88 for real-time application. The application was mainly designed for Futronics FS88 Scanner with 500dpi fingerprint image with resolution of $320 * 480$ Pixels,.

The fingerprint data using FS88 scanner was collected from 60 individuals per subject 10 fingerprints from two thumbs. For testing the database was unconstrained, i.e. it contained normal, dry \& wet fingerprints. The fingerprints were of all type, having clear core as well as without core, having arch or high curvature region. Overall the conditions were random. One extra set is created for fingerprints containing clear core point as shown in Figure 11 (b). Total 200 tests were performed on collected fingerprints and the results are as follows.

Table 1. Test Results

\begin{tabular}{|l|l|r|l|}
\hline & \multicolumn{1}{|l|}{$\begin{array}{l}\text { FS 88 } \\
\text { Parameter }\end{array}$} & $\begin{array}{l}\text { FVC } \\
\mathbf{2 0 0 2 , 2 0 0} \\
\text { Database }\end{array}$ & $\begin{array}{l}\text { Fingerprints } \\
\text { with clear } \\
\text { Core point }\end{array}$ \\
\hline \% Accuracy & 84 & 68 & 98 \\
\hline $\begin{array}{l}\text { Average Error } \\
\text { (Pixels) }\end{array}$ & 5.57 & 6.13 & 2.50 \\
\hline $\begin{array}{l}\text { Average Execution } \\
\text { Time (ms) }\end{array}$ & 16.41 & & 16 \\
\hline
\end{tabular}

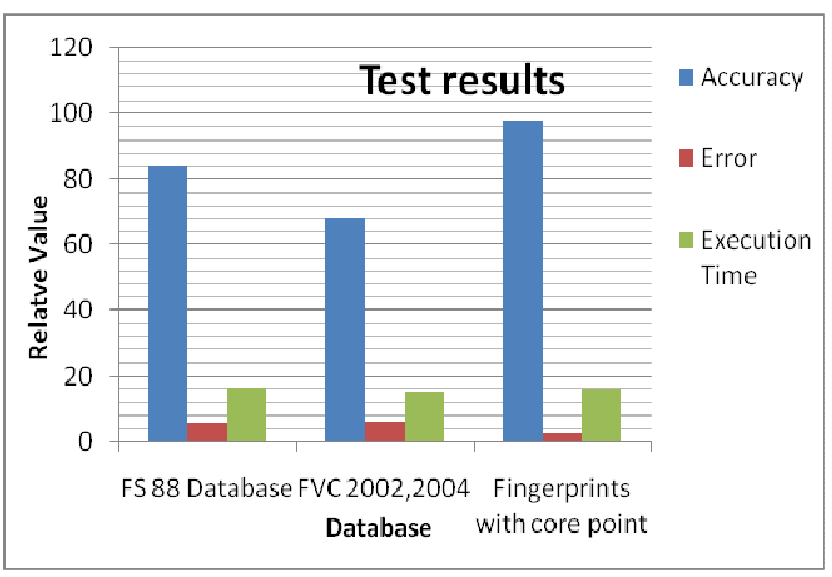

Figure 12 . Test Results for FS 88 \& FVC Databases

All fingerprints do not have core and hence to decide the reference point other methods are suggested in [8][14] base on covariance matrix of the orientation field. We consider both the condition. On the random database the achieved error is $84 \%$ on FS88 database and that with the FVC database is $68 \%$ as these database have extreme conditions fingerprints and the varying image size for FVC 2002 \& FVC 2004 database images.

The results are improved when fingerprint containing clear core points were considered and for such database we have achieved $98 \%$ accuracy and average error (Distance from actual core point) as 2.5 Pixels, which is 5.55 \& 6.13 pixels for FS88 \& FVC 
database respectively. The average execution is around $16 \mathrm{~ms}$ for all the databases.

\section{CONCLUSION}

In this paper we have proposed an algorithm to detect a reference point also referred as singular point for development of correlation based automatic fingerprint recognition systems. The main aim is to detect core point (loops), the algorithm detects clear core point with $98 \%$ accuracy. The overall accuracy on unconstrained database is $84 \%$. This can be improved by adding more heuristics and alternative mechanism to detect the reference point if core or arch type of singularity is not present on the finger. The proposed algorithm successfully detects singularity like Core Point (loop) and Arch and can be used in reference point detection step for the correlation based Automatic Fingerprint recognition System

\section{REFERENCES}

[1] Afsar F., Arif M. and Hussain M.(2004) , Fingerprint Identification and Verification System using Minutiae Matching , In Proceedings of National Conference on Emerging Technologies, Pakistan Institute of Engineering \& Applied Sciences, Islamabad, Pakistan

[2] Cavusoglu A., Gorgunoglu S.(2007), A Robust Correlation based Fingerprint Matching Algorithm for Verification, Journal of Applied Science, Vol 7, Asian network for Scientific Information : ISSN : 1812-5654

[3] C. V. Kameswara Rao and K. Balck, " Finding The Core Point In A Fingerprint", IEEE Transactions on Computers, Vol. C-27, No. 1, January 1978

[4] Fingerprint verification Competition 2006, [WWW document] http://bias.csr.unibo.it/fvc2006, (Accessed 25 ${ }^{\text {th }}$ March 2009)

[5] Hong L., Jain A., Bolle R. (1997), On-Line Fingerprint Verification, IEEE: Transaction on Pattern Analysis and Machine Intelligence, Vol. 19, No. 4

[6] Hong L., Jain A. (1998), Fingerprint Image Enhancement : Algorithm and Performance Evaluation, IEEE: Transaction on Pattern Analysis and Machine Intelligence, Vol. 20, No. 8.

[7] Jain A., Hong L.(2000), A Multichannel Approach for Fingerprint Classification ,In IEEE Transactions On Image Processing, Vol. 9, No. 5.
[8] Jain A., Prabhakar S., Hong L, and Pankanti S.(2000), Filter bank-Based Fingerprint Matching, IEEE: Transactions On Image Processing, Vol. 9, No. 5 .

[9] Kekre H. B., Bharadi V.A. (2009), Fingerprint Orientation Field Estimation Algorithm Based on Optimized Neighborhood Averaging, 2nd International Conference on Emerging Trends in Engineering \& Technology (Accepted), Nagpur ,India.

[10] Kekre H. B., Bhatnagar S. ,Finger Print Matching Techniques, In Proceedings of National Conference on Applications Digital Signal Processing. (NCDSP - 2007), Mumbai, Jan 19 - 20, 2007.

[11] Kekre H., Sarode T., Rawool V. (2008) , Finger Print Identification using Discrete Sine Transform (DST)", In Proceedings International Conference on Advanced Computing \& Communication Technology (ICACCT-2008), Asia Pacific Institute of Information Technology, Panipat India.

[12] Kekre H., Sarode T., Thepade S. (2008), DCT Applied to Column Mean and Row Mean Vectors of Image for Fingerprint Identification, In Proceedings International Conference on Computer Networks and Security (ICCNS08), Pune, India .

[13] Maio D., Maltoni D. et. al. (2000), FVC2000: Fingerprint Verification Competition, Report of FVC 2000 .

[14] Maltoni ,D., Maio D., Jain A, and Prabhakar S.(2003), Handbook of Fingerprint Recognition, Springer-Verlag.

[15] S. Chikkukerurr, N. Ratha, "Impact of Singular Point Detection on Fingerprint Matching Performance", autoid, pp.207-212, Fourth IEEE Workshop on Automatic Identification Advanced Technologies (AutoID'05), 2005

[16] S. Chikkukerur, A. Kartwrieght, V. Govindrajalu, "Fingerprint Image Enhancement using STFT Analysis", AutoID 2005: 139-143 ICAPR (2) 2005: 20-29

[17] Wang Y., Hu J., Han F. (2007), Enhanced Gradient Based Algorithm for the Estimation of Fingerprint Orientation Field, In Proceedings: Applied Mathematics and Computation 185(2007) 823-833, Science Direct: Elsevier.

[18] Woodward, J. ,Orlans, P. , Higgins T.(2003), Biometrics, McGraw-Hill/Osborne. 